\title{
The glymphatic system and its relation with neurological diseases
}

\author{
Karla Helena Picoli Natário ${ }^{1}$ (D) Guilherme Brasileiro de Aguiar ${ }^{2 *}$ (D), \\ Marcelo Adriano da Cunha e Silva Vieira ${ }^{3}$ (1)
}

\section{INTRODUCTION}

The glymphatic system (GS) is a paravascular pathway located between the vascular adventitia and vascular astrocytic end-feet. It is responsible for clearing toxic peptides from the cerebral parenchyma ${ }^{1,2}$. This paravascular clearance system was named glymphatic system due to the fusion of the words "glial" and "lymphatic" since its very existence depends on glial cells and also because of its functional similarity with the peripheral lymphatic system ${ }^{1}$.

Jessen et al. ${ }^{2}$ have demonstrated a relationship between a dysfunctional GS and the emergence of neurodegenerative diseases, as well as situations of excessive sleep deprivation and natural aging ${ }^{2,3}$. Several neurodegenerative diseases have been associated with these conditions. Alzheimer's dementia (AD) is the main target of current research ${ }^{2,3}$. Despite the literature limitations on the study of the GS, new correlations with neurodegenerative diseases are constantly emerging.

This article aims to review how the glymphatic system functions, the factors that interfere with it, and its correlation with neurological pathologies.

\section{METHODOLOGY}

This is a descriptive study based on the literature available in the MEDLINE/Pubmed database. The terms searched were the following: "glymphatic system," AND "sleep," "cognitive decline," "aging," "neurodegenerative disease," "Alzheimer's disease”, all in English. All articles considered relevant were included in this review, as were the studies referenced therein, in order to raise awareness about the method. Duplicate items were discarded.

\section{DISCUSSION}

Approximately $68 \%$ of the central nervous system's total volume of water is in the intracellular space, whereas the remaining $32 \%$ is in the extracellular space ${ }^{1}$. The extracellular space is distributed in the interstitial fluid (ISF), cerebrospinal fluid (CSF), and cerebral blood circulation ${ }^{1,2}$. The CSF accounts for approximately $10 \%$ of the total volume of intracranial fluid ${ }^{4}$, being produced by the choroid plexuses and playing an important role in the distribution of nutrients and removal of toxic interstitial metabolites ${ }^{2}$. The CSF, after circulating through the ventricular system and subarachnoid space of the cortex and spinal $\operatorname{cord}^{2}$, penetrates into a perivascular space called the Virchow-Robin space ${ }^{2}$.

The Virchow-Robin spaces are filled with CSF and bounded by a leptomeningeal cell layer on the inner wall - facing the vessel, and on the outer wall, facing the perivascular astrocytic end-feet ${ }^{2,5}$. The central nervous system (CNS) has all its blood vessels surrounded by vascular astrocytic end-feet ${ }^{2}$. These vascular end- feet create the outer wall in the perivascular space, resembling a tunnel that surrounds the vasculature ${ }^{2}$.

From the subarachnoid space, the CSF is directed to the Virchow-Robin spaces by a combination of arterial pulsatility, breathing, and pressure gradients ${ }^{2}$. The CSF and ISF exchange continuously due to the continuous influx of CSF into the perivascular spaces ${ }^{2}$. The subsequent distribution of the interstitial fluid to the brain parenchyma is facilitated by aquaporin- 4 (AQP4) water channels ${ }^{2}$, with this protein being expressed in the polarized portion of astrocytic end-feet. In these, the CSF penetrates into the parenchyma along the paravascular spaces

\footnotetext{
'Universidade Municipal de São Caetano do Sul - São Paulo (SP), Brazil.

${ }^{2}$ Faculdade de Ciências Médicas da Santa Casa de São Paulo - São Paulo (SP), Brazil.

${ }^{3}$ Instituto de Doenças Tropicais Natan Portela - Teresina (PI), Brazil.

*Corresponding author: guilhermebraguiar@yahoo.com.br

Conflicts of interest: the authors declare there are no conflicts of interest. Funding: none.

Received on December 22, 2020. Accepted on January 03, 2021.
} 
that surround the penetrating arteries, and the interstitial cerebral fluid is drained along the paravenous pathways ${ }^{1,2}$ (Figure 1).

This elimination pathway and the role of AQP4 channels in the clearance of neurotoxic metabolites were demonstrated in 2012 by Iliff et al. ${ }^{6}$, in a study based on an in vivo two-photon imaging technique with small fluorescent mark$\mathrm{ers}^{5}$. Animals without astrocytic AQP4 channels have been shown to exhibit a $70 \%$ reduction in interstitial solute clearance ${ }^{5}$. Thus, one can infer that substances involved in neurodegenerative pathologies could accumulate inadequately in the glymphatic pathway ${ }^{3,5}$.

Even though the CSF and ISF that drain into the subarachnoid space exit the skull through unidirectional arachnoid granulations, Lee et al. ${ }^{7}$ have suggested that part of that CSF follows along the internal carotid artery through the perivascular spaces, as well as within the perineural spaces of the cranial nerves, notably the olfactory and vagus nerves ${ }^{4,7}$. Noteworthy are the extensions that follow the olfactory tracts across the cribriform plate and protrude into the nasal submucosa. They have been shown to account for $15-30 \%$ of CSF solute removal ${ }^{4}$. The nasal submucosa has a dense lymphatic network that drains the CSF and solutes into the deep cervical lymph nodes and is of special importance in the removal of molecules with a large molecular weight ${ }^{2,4}$.

In addition to the aforementioned physiological variations, some other factors interfere with the glymphatic influx, such as arterial pulsatility, sleep, and the natural aging process.

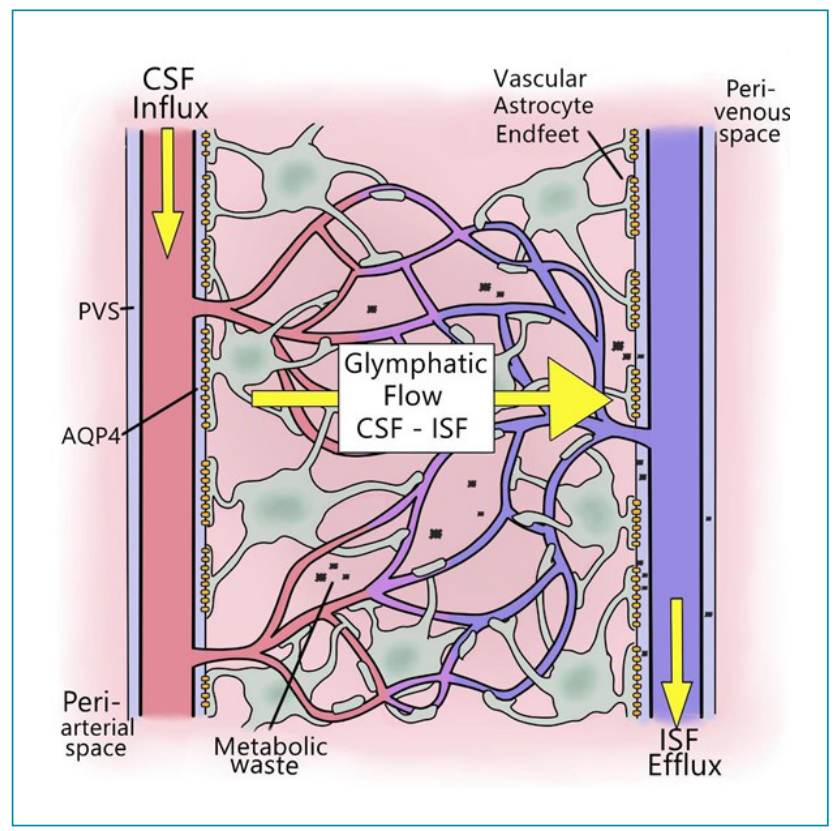

Figure 1. Schematic representation of the encephalic lymphatic flow

\section{Arterial pulsatility}

The constant production of CSF by the choroid plexus creates a pressure that determines the direction of fluid flow through the ventricular system into the subarachnoid space $^{2}$. The CSF entering the perivascular space is essential for facilitating both lymphatic exchange and clearance ${ }^{2}$. The CSF has already been shown to follow the course of cerebral arteries, especially due to the pulsatility generated by smooth muscle cells that create pulse waves along the entire length of penetrating pial arteries, which explains why the perivascular influx occurs preferentially around the arteries rather than in brain veins ${ }^{2,4}$.

With the aid of ultra-fast magnetic resonance imaging (MRI) encephalography, it was possible to reveal that pulsations related to the respiratory cycle and changes in the vasomotor tone also propagate through the human brain and, therefore, can potentially contribute to the glymphatic $\mathrm{flow}^{2,3}$. On the other hand, the CSF flow within the ventricular compartments can be largely driven by breathing, which is also considered an important factor in the glymphatic flow ${ }^{2}$.

\section{Sleep}

Sleep has been identified as one of the main factors interfering with the dynamics of glymphatic influx. Recent studies have shown that sleep is the only state during which glymphatic activity is active, suggesting that it is suppressed during wakefulness ${ }^{2,8}$. Such findings indicate that the sleep state is particularly conducive to the convective flow of fluids and, therefore, to the release of metabolites ${ }^{2,8}$. In this way, sleep seems to increase glymphatic activity and, consequently, the removal of neurotoxic waste produced during wakefulness ${ }^{2,8}$.

This feature was demonstrated in 2013 by Xie et al. ${ }^{8}$, by comparing in vivo two-photon images of the CSF flow in the cortex of awake, anesthetized, and sleeping mice ${ }^{8}$. Anesthetized and sleeping mice had a $60 \%$ increase in the interstitial volume and rate of $\beta$-amyloid clearance during sleep ${ }^{8}$. Conversely, while the mice were awake, there was a reduction in the volume of the interstitial space and, consequently, an increase in convective fluid resistance and CSF flow suppression ${ }^{2,8}$. The activity of neuromodulators, such as glutamate and noradrenaline, has been linked to a reduction in interstitial space, especially noradrenergic signaling originating from the locus ceruleus ${ }^{8}$. These modify the cell volume and thus reduce the size of the interstitial space, which prevents the CSF influx observed during sleep ${ }^{8}$.

\section{Aging}

Aging is a major contributor to decreased glymphatic activity ${ }^{2}$. Jessen et al. ${ }^{2}$ evaluated the glymphatic function of young and elderly mice and demonstrated the occurrence of a significant reduction in this function in approximately $80-90 \%$ of 
the older mice. This is mainly due to a change in the location of the AQP4 enzymes in young animals, which remain in the astrocytic end-feet and play a fundamental role in interstitial solute clearance through the perivascular drainage pathways ${ }^{2,9}$. In elderly animals, these enzymes lose their perivascular polarization and are concentrated in the astrocytic parenchymal processes, thus impairing the exchange of CSF and ISF, leading to a decline in glymphatic activity as age advances?

Other factors that can contribute to a decreased activity of the GS are a decline in CSF production (66\%) and pressure $(27 \%)^{2}$, in addition to arterial stiffening resulting from aging, which reduces arterial pulsatility and, consequently, decreases glymphatic influx ${ }^{2}$. The decline in glymphatic activity associated with advancing age is a fundamental condition for the emergence of neurological disorders ${ }^{2,4}$. It should be noted that most neurodegenerative diseases have aging as a common risk factor ${ }^{2}$. A failure in the lymphatic system with progressive aging can lead to the accumulation of aggregated and hyperphosphorylated proteins, which makes the senile brain more vulnerable to the development of neurodegenerative pathologies and even to cognitive dysfunction ${ }^{2,9}$.

\section{The Glymphatic System and neurodegenerative diseases}

Most neurodegenerative diseases are characterized by the accumulation of aggregated proteins in the $\mathrm{CNS}^{2}$ (Table 1). These protein aggregates are present in both the ISF and CSF, as are $\beta$-amyloid and tau folded fibrillary tangles in $\mathrm{AD}$, and folded $\alpha$-synuclein in Parkinson's disease ${ }^{2}$.

$\mathrm{AD}$ is characterized by the accumulation of $\beta$-amyloid protein and the formation of tau substance tangles in various brain regions ${ }^{2,4}$. Impaired clearance of these substances by the GS favors the accumulation of $\beta$-amyloid in the cerebral parenchyma and disease progression ${ }^{1,2}$. This deregulation is a consequence of changes in the expression of AQP4 that occur in aging and the breakdown of the blood-brain barrier, which contributes to the accumulation of $\beta$-amyloid in the cerebral vasculature ${ }^{1,3}$, resulting in cerebral amyloid angiopathy ${ }^{10}$, which consequently will culminate in arterial stiffening and impaired glymphatic flow ${ }^{10}$.

Rassmussen et al. ${ }^{9}$ and Reddy et al. ${ }^{11}$ demonstrated in an animal model with $\mathrm{AD}$ that this change in the expression of AQP4, both in its erratic location and in the loss of polarization,

Table 1. Some diseases and their respective mechanisms of glymphatic system dysfunction.

\begin{tabular}{|c|c|c|}
\hline Authors & $\begin{array}{l}\text { Disease associated with } \\
\text { a dysfunction in the } \\
\text { glymphatic system }\end{array}$ & Clinical and pathological impairment \\
\hline $\begin{array}{l}\text { Peng et al. }{ }^{12} \\
\text { (2016). }\end{array}$ & Alzheimer's disease & $\begin{array}{c}\text { Aging associated with a dysfunctional blood-brain barrier and } \\
\text { dysfunctional AQP4 enzymes implies glymphatic dysfunction, which } \\
\text { promotes the accumulation of protein aggregates ( } \beta \text {-amyloid and tau } \\
\text { substance) with a more accelerated disease progression. }\end{array}$ \\
\hline $\begin{array}{l}\text { Prasad et al. }{ }^{14} \\
\text { (2014). }\end{array}$ & Diabetes & $\begin{array}{l}\text { Hyperglycemia, formation of ROS, blood-brain barrier dysfunction, } \\
\text { and abnormal neovascularization lead to losses in the drainage of } \\
\text { neurotoxic solutes from the interstitial fluid by the glymphatic pathway, } \\
\text { favoring the development of neurodegenerative diseases. }\end{array}$ \\
\hline $\begin{array}{l}\text { \|liff et al. }{ }^{3} \\
\text { (2013). }\end{array}$ & Vascular dementia & $\begin{array}{l}\text { Structural changes in the cerebral blood vessels resulting mainly from } \\
\text { atherosclerosis impair the drainage of neurotoxic solutes through the } \\
\text { glymphatic route, which favors the appearance of dementia. }\end{array}$ \\
\hline $\begin{array}{l}\text { lliff et al. } .^{15} \\
\text { (2014). }\end{array}$ & Traumatic injury & $\begin{array}{l}\text { The formation of astroglial scars, neuroinflammation, and the impaired } \\
\text { expression of AQP4 disrupt glymphatic function, which contributes to } \\
\text { an increase in neuronal damage secondary to traumatic injury and the } \\
\text { emergence of neurodegenerative pathologies. }\end{array}$ \\
\hline $\begin{array}{l}\text { Zou et at. }{ }^{16} \\
2019 .\end{array}$ & Parkinson's disease & $\begin{array}{l}\text { The perivascular accumulation of } \alpha \text {-synuclein aggregates and a change } \\
\text { in AQP4 expression in the substantia nigra and in the autophagy of this } \\
\text { substance caused neuroinflammation, neuronal damage, and errors in } \\
\text { glymphatic function, which demonstrated progression of PD. }\end{array}$ \\
\hline $\begin{array}{l}\text { Jessen et al. }{ }^{2} \\
\text { (2015). }\end{array}$ & $\begin{array}{l}\text { Amyotrophic lateral sclerosis } \\
\text { and frontotemporal dementia }\end{array}$ & $\begin{array}{c}\text { An increase in the levels of noradrenaline in the CSF may lead to a } \\
\text { reduction in glymphatic activity and AQP4 channels, impairing this } \\
\text { neurotransmitter's glymphatic function and worsening the clinical } \\
\text { progression of the pathologies. }\end{array}$ \\
\hline
\end{tabular}

AQP4: aquaporin 4; ROS: reactive oxygen species; PD: Parkinson's disease; CSF: cerebrospinal fluid. 
modified the glymphatic clearance, favoring the accumulation of $\beta$-amyloid in the brain ${ }^{9,11}$.

Peng et al. ${ }^{12}$, in a study published in 2016, demonstrated the erratic deposit of $\beta$-amyloid in the perivascular spaces of transgenic mice based on the expression of the human amyloid precursor protein. Changes in the clearance of this substance have become evident due to reduced receptors in the blood-brain barrier ${ }^{12}$. Accordingly, it is important to note that significant deposits of $\beta$-amyloid were preceded by lymphatic failure, which therefore can be an early biomarker of $\mathrm{AD}^{12}$. Jessen et al. ${ }^{2}$ also demonstrated that the APOE gene, responsible for expressing apolipoproteins for cerebral lipid transport, is related to the elimination of $\beta$-amyloid ${ }^{2,13}$. Thus, defects in its gene expression can be considered relevant genetic risk factors for $\mathrm{AD}$ and impaired glymphatic function ${ }^{2,13}$.

Similar to $\mathrm{AD}$, vascular dementias are also influenced by the GS. Changes in the structure of blood vessels due to high blood pressure and atherosclerosis may damage the perivascular space and impair drainage of toxic solutes by the $\mathrm{GS}^{1,3}$. It is speculated that an abnormal increase in the perivascular space in these diseases may have an impact on solute elimination flows, resulting in interstitial fluid obstruction, favoring the deposition of neurotoxic substances and, consequently, the appearance of degenerative pathologies ${ }^{1,3}$.

Recently, diabetes has been linked to vascular complications and neurodegenerative diseases, such as $\mathrm{AD}^{1,14}$. Neuronal damage caused by hyperglycemia and the formation of reactive oxygen species affect the blood-brain barrier function, in addition to causing abnormal vascular remodeling ${ }^{14}$. These changes promote inappropriate clearance of the interstitial solute and other substances, such as $\beta$-amyloid, through the glymphatic pathway, favoring the accumulation of these toxic compounds and the development of pathologies in the $\mathrm{CNS}^{1,14}$.

Like aging, traumatic brain injury can lead to progressive neurodegeneration and induce the release of peptides, such as C-tau ${ }^{15}$. This is a biomarker of brain injury and correlates with the severity of traumatic injury ${ }^{15}$. This pathology is linked to the formation of astroglial scars and persistently activated neuroinflammation ${ }^{4,9}$. As a result, there is a marked decrease in glymphatic function and changes in the expression of AQP4. This reinforces the important role the GS plays in eliminating toxic solutes into the interstitial fluid ${ }^{4,9}$, an association between dysfunction in this system and progressive neurological involvement in patients with traumatic brain injury.

In 2014, Iliff et al. ${ }^{15}$ tracked the pathway of human tau release with the aid of intracortical injections in in vivo models. It was seen that large amounts of human tau were deposited around blood vessels, which hindered the removal of this protein by the glymphatic pathway, thereby aggravating neuronal damage secondary to traumatic injury ${ }^{15}$.

Another neurodegenerative disease involving dysfunctional processes in the GS is Parkinson's disease (PD ${ }^{2,16}$. PD is characterized by the progressive loss of dopaminergic neurons in the midbrain (substantia nigra) and the formation of Lewy bodies ${ }^{2,16}$. The pathophysiological mechanism of PD involves an imbalance between the production and release of $\alpha$-synuclein in the brain due to mutations in the $\alpha$-syn gene and a decreased removal rate of this compound due to pathogenesis mechanisms still poorly elucidated in the literature ${ }^{16}$. Zou et al. ${ }^{16}$ demonstrated the perivascular accumulation of $\alpha$-synuclein aggregates and changes in the expression of AQP4 in the black substance by blocking the lymphatic drainage in young mice, which resulted in neuroinflammation with dopaminergic neuronal loss and motor deficits ${ }^{16}$. Furthermore, it became evident that changes in the autophagy process of $\alpha$-synuclein favor the formation of aggregates of the substance and impair the flow of lymphatic clearance, worsening the progression of the pathology in the mice studied ${ }^{16}$.

In addition to the aforementioned conditions, amyotrophic lateral sclerosis (ALS) and frontotemporal dementia (FTD) are related to a dysfunctional GS and progression of the neurological scenario $^{17,18}$. ALS and FTD are multisystem neurodegenerative diseases with progressive neurological deterioration ${ }^{17}$. Approximately $50 \%$ of individuals with ALS have FTD ${ }^{18}$. Recently, the GS has been shown to participate in the pathophysiology of ALS and DFT, having been associated with inefficient clearance of neurotoxic substances ${ }^{17}$. Brooks et al. ${ }^{19}$ found that individuals with ALS and DFT have high noradrenaline levels in their CSF and neuronal tissues ${ }^{17,79}$. Jessen et al. ${ }^{2}$, in 2015 , hypothesized that increased noradrenaline in the CSF might lead to a reduction in glymphatic activity, thereby impairing AQP4 channels and thus creating a turbulent interstitial flow. All this would in turn cause harm to the glymphatic function of this neurotransmitter, worsening the dementia process involved in these pathologies ${ }^{3,17}$ (Table 1).

\section{Limitations of the study}

The main limitation of this study is its narrative nature, therefore, not following systematic evidence-based criteria.

\section{CONCLUSION}

The glymphatic system plays an important role in the elimination of neurotoxic peptides and is closely related to the development of neurodegenerative diseases, most of which are secondary to the erratic accumulation of those substances. However, further studies are still needed to find out how the glymphatic flow functions, especially through radiological techniques, such as magnetic resonance imaging, and biomarkers, for the early 
detection of cellular changes and also to propose appropriate therapeutic interventions. In the future, a complete understanding of the GS may help in the prevention of degenerative diseases of the central nervous system.

\section{AUTHORS" CONTRIBUTIONS}

KHPN: Conceptualization, Data Curation, Writing - Original Draft. GBA: Conceptualization, Writing - Original Draft. MACSV: Conceptualization, Writing - Review \& Editing.

\section{REFERENCES}

1. Kim YK, Nam KI, Song J. The Glymphatic System in DiabetesInduced Dementia. Front Neurol. 2018;9:867. https://doi. org/10.3389/fneur.2018.00867

2. Jessen NA, Munk AS, Lundgaard I, Nedergaard M. The glymphatic system: a beginner's guide. Neurochem Res. 2015;40(12):258399. https://doi.org/10.1007/s11064-015-1581-6

3. Iliff JJ, Lee H, Yu M, Feng T, Logan J, Maiken Nedergaard M. et al. Brain-wide pathway for waste clearance captured by contrast-enhanced MRI. J Clin Invest. 2013;123(3):1299-309. https://doi.org/10.1172/JCl67677

4. Plog BA, Nedergaard M. The glymphatic system in central nervous system health and disease: past, present, and future. Annu Rev Pathol. 2018;13:379-94. https://doi.org/10.1146/ annurev-pathol-051217-111018

5. lliff JJ, Nedergaard M. Is there a cerebral lymphatic system? Stroke. 2013;44(6 Suppl 1):S93-5. https://doi.org/10.1161/ STROKEAHA.112.678698

6. Iliff JJ, Wang M, Liao Y, Plogg BJ, Peng W, Gundersen GA, et al. A paravascular pathway facilitates CSF flow through the brain parenchyma and the clearance of interstitial solutes, including amyloid $\beta$. Sci Transl Med. 2012;4(147):147ra111. https://doi.org/10.1126/scitranslmed.3003748

7. Lee $H$, Xie L, Yu M, Kang $H$, Feng $T$, Deane $R$, et al. The Effect of Body Posture on Brain Glymphatic Transport. J Neurosci. 2015;35(31):11034-44. https://doi.org/10.1523/ JNEUROSCI.1625-15.2015

8. Xie L, Kang H, Xu Q, Chen MJ, Liao Y, Thiyagarajan M, et al. Sleep drives metabolite clearance from the adult brain. Science. 2013;342(6156):373-77. https://doi.org/10.1126/ science. 1241224

9. Rasmussen MK, Mestre H, Nedergaard M. The glymphatic pathway in neurological disorders. Lancet Neurol. 2018;17(11):1016-24. https://doi.org/10.1016/S1474-4422(18)30318-1

10. Reeves BC, Karimy JK, Kundishora AJ, Mestre H, Cerci HM, Matouk C, et al. Glymphatic system impairment in alzheimer's disease and idiopathic normal pressure hydrocephalus. Trends Mol Med. 2020;26(3):285-95. https://doi.org/10.1016/j. molmed.2019.11.008
11. Reddy OC, van der Werf YD. The sleeping brain: harnessing the power of the glymphatic system through lifestyle choices. Brain Sci. 2020;10(11):868. https://doi.org/10.3390/brainsci10110868

12. Peng $W$, Achariyar TM, Li B, Liao Y, Mestre H, Hitomi E, et al. Suppression of glymphatic fluid transport in a mouse model of Alzheimer's disease. Neurobiol Di. 2016;93:215-25. https:// doi.org/10.1016/j.nbd.2016.05.015

13. Xu Q, Bernardo A, Walker D, Kanegawa T, Mahley RW, Huang Y. Profile and regulation of apolipoprotein $\mathrm{E}$ (ApoE) expression in the CNS in mice with targeting of green fluorescent protein gene to the ApoE locus. J Neurosci. 2006;26(19):4985-94. https://doi.org/10.1523/JNEUROSCI.5476-05.2006

14. Prasad S, Sajja RK, Naik P, Cucullo L. Diabetes Mellitus and Blood-Brain Barrier Dysfunction: An Overview. J Pharmacovigil. 2014;2(2):125. https://doi.org/10.4172/2329-6887.1000125

15. Iliff JJ, Chen MJ, Plog BA, Zeppenfeld DM, Soltero M, Yang $L$, et al. Impairment of glymphatic pathway function promotes tau pathology after traumatic brain injury. J Neurosci. 2014;34(49):16180-93. https://doi.org/10.1523/ JNEUROSCI.3020-14.2014

16. Zou W, Pu T, Feng W, Lu M, Zheng Y, Du R, et al. Blocking meningeal lymphatic drainage aggravates Parkinson's diseaselike pathology in mice overexpressing mutated $\alpha$-synuclein. Transl Neurodegener. 2019;8:7. https://doi.org/10.1186/ s40035-019-0147-y

17. Radford RA, Morsch M, Rayner SL, Cole NJ, Pountney DL, Chung RS. The established and emerging roles of astrocytes and microglia in amyotrophic lateral sclerosis and frontotemporal dementia. Front Cell Neurosci. 2015;9:414. https://doi. org/10.3389/fncel.2015.00414

18. Teixeira-Jr AL, Salgado JV. Demência fronto-temporal: aspectos clínicos e terapêuticos. Rev Psiquiatr Rio Gd. do Sul. 2006;28(1):69-76. https://doi.org/10.1590/S010181082006000100009

19. Brooks BR, Zielger MG, Lake CR, Wood JH, Enna SJ, Engel WK. Cerebrospinal fluid norepinephrine and free $\gamma$-aminobutyric acid in amyotrophic lateral sclerosis. Brain Res Bull. 1980;5(Suppl 2):765-8. https://doi.org/10.1016/0361-9230(80)90126-4 